\title{
Design, synthesis, spectral characterization and bioactivity evaluation of new sulfonamide and carbamate derivatives of 5- Nitro-1H-indazole
}

\author{
K. Pushpa Kumar ${ }^{\oplus 1}$, Pandreti Vedavathi $^{\oplus 2}$, K. Venkata Subbaiah ${ }^{\oplus 3}$, \\ D. Venkata Ramana Reddy ${ }^{\oplus 1}$ and C. Naga Raju ${ }^{\oplus *}$
}

${ }^{I}$ Department of Chemistry, S. G. Govt. Degree \& PG College, Pileru, Sri Venkateswara University, Tirupati-517502, A.P., India

${ }^{2}$ Department of Chemistry, Sri Venkateswara University, Tirupati-517502, A.P., India

${ }^{3}$ DST Purse Centre, Sri Venkateswara University, Tirupati-517502., A.P., India

(Received May 16, 2017; Revised September 09, 2017; Accepted September 10, 2017)

\begin{abstract}
A series of new sulfonamide and carbamate derivatives of 5-nitro- $1 H$-indazole was synthesized in good yields. The structures of all the newly synthesized compounds were confirmed by $\mathrm{IR},{ }^{1} \mathrm{H},{ }^{13} \mathrm{C}-\mathrm{NMR}$, Mass spectra and elemental analysis. The compounds $\mathbf{9 a}$ and $\mathbf{9 f}$ showed high antibacterial activity against $L$. bacillus and $S$. aureu, $9 \mathbf{f}$ and $\mathbf{9 j}$ exhibited good activity against $E$. coli and $P$. florescensa, whereas the compounds $9 \mathbf{a}$, 9d. 9f and 9j showed good antifungal activity against tested fungal strains, however, compared to all the compounds $9 \mathbf{e}$ and $9 \mathbf{h}$ exhibited significant activity against $A$. niger and $P$. chrysogenum respectively.
\end{abstract}

Keywords: 5-Nitroindazole; sulfonamides; carbamates; antimicrobial activity. C2017 ACG Publications. All rights reserved.

\section{Introduction}

In spite of versatile applications of indazole derivatives, its biological applications were not explored compared to other hetero arenes, indole and imidazoles. Indazole differs from indole by an additional ring nitrogen consequently displays promising pharmacological activity. Indazole derivatives have aroused great interest due to their wide range of biological importance. Indazole derivatives possess pharmacological activities, ${ }^{1}$ such as antihelmintic, ${ }^{2}$ antihistaminic, ${ }^{3}$ antimicrobial, ${ }^{4}$ antipsychotic, ${ }^{5}$ antiepileptic, anti-depressant, ${ }^{6}$ anti-inflammatory, ${ }^{7,8}$ analgesic and antipyretic, ${ }^{9}$ dopamine antagonistic, ${ }^{10}$ anti-tumor ${ }^{11}$ and anti-HIV activities. ${ }^{12}$ In the previous research, indazole

\footnotetext{
* Corresponding author: E-mail: rajuchamarthi10@gmail.com Phone: +91 - 9703193375
} 
derivatives are known to be active and have important pharmacological properties like cancer cell proliferative disorders, Alzheimer's disease, protein kinase inhibitors and also act as an antiviral agents. ${ }^{13-15}$ Indazoles have been showing potential biological activities that are enhanced by substitution/addition of different functional groups which have been illustrated in the literature survey.

In addition, sulfonamide and carbamate derivatives have a wide range of biological applications. Sulfonamide derivatives are an important class of drugs $\left(-\mathrm{SO}_{2}-\mathrm{N}-\right)$ which are bacteriostatic agents and find use in both human therapy and animal husbandry. ${ }^{16}$ Sulfonamides exhibit different types of pharmacological activities such as antitumor, ${ }^{17}$ hypoglycemic,${ }^{18}$ antithyroid, ${ }^{19}$ anticarbonicanhydrase,${ }^{20}$ anti-inflammatory, ${ }^{21}$ diuretic, ${ }^{22} \mathrm{COX}$ inhibitors, the enzyme dihydropteroatesynthetase (DHPS)-the key enzyme involved in folate synthesis), anti-impotent drugs, ${ }^{23}$ and anti-glaucoma. ${ }^{24-25}$ Sulfonamides act as protease inhibitors which are used to treat HIV infection (Amprenavir, 1), prevent and reduce the symptoms of altitude sickness (Acetazolamide, 2) and used to cure severe burns (Mafenide, 3). Carbamate derivatives are found in agricultural chemicals, such as fungicides (Idopropynylbutylcarbamate 4), pesticides (Carbaryl, 5) and herbicides. Organic carbamates are expensive synthetic intermediates and establish in a diversity of biologically active compounds. ${ }^{26}$ Based on the importance of this class of biologically active compounds, the synthesis of carbamates is a high-value endeavour.

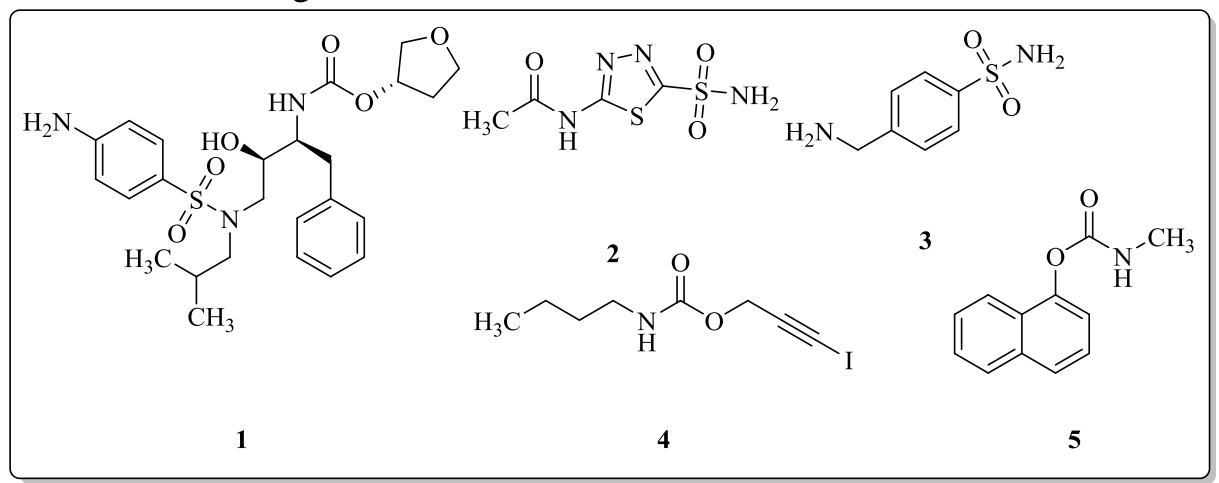

Figure 1. Drug Molecules containing sulfonamide and carbamate moieties.

Our research is focused on the insertion/substitution, within the 5-nitroindazole molecule, by different chemically reactive groups such as substituted chloroformates and sulfonylchlorides. The synthesized compounds were screened for their antibacterial and antifungal activities.

\section{Results and Discussions}

\subsection{Chemistry}

The synthetic method adopted for the synthesis of sulfonamide and carbamate derivatives of 5-nitro- $1 H$-indazole is outlined in Scheme 1.

The 5-nitro- $1 H$-indazole (6) was reacted with $\mathrm{NaH}$ in dry tetrahydrofuran at 5-30 ${ }^{\circ} \mathrm{C}$ to obtain the sodium salt of 5-nitro-1H-indazole (7) intermediate and hydrogen gas is evolved. Further, the intermediate was reacted with various sulfonyl chlorides 8a-e and chloroformates $\mathbf{8 f}-\mathbf{j}$ at $10-50{ }^{\circ} \mathrm{C}$ to afford 1-(substituted sulfonyl)-5-nitro- $1 H$-indazole 9a-e and 5-nitro- $1 \mathrm{H}$-indazole-1-carboxylates 9f-j in high yields. The progress of the reaction was monitored by TLC. After completion of the reaction, it was filtered to remove sodium chloride and the solvent was removed under reduced pressure to obtain a crude product. It was purified by column chromatography using silica gel (60-120 mesh) as adsorbent and hexane and ethyl acetate mixture (1:2) as an eluent to obtain pure title compounds 9a-e and $\mathbf{9 f}-\mathbf{j}$. All the sulfonamide derivatives 9a-e of this series was prepared using the same procedure by reacting various sulfonyl chlorides 8a-e with compound 7. Similarly, carbamate derivatives $\mathbf{9 f - j}$ were also prepared by reacting various alkyl/aryl chloroformates $\mathbf{8 f}-\mathbf{j}$ with $\mathbf{7}$ using the above procedure. 


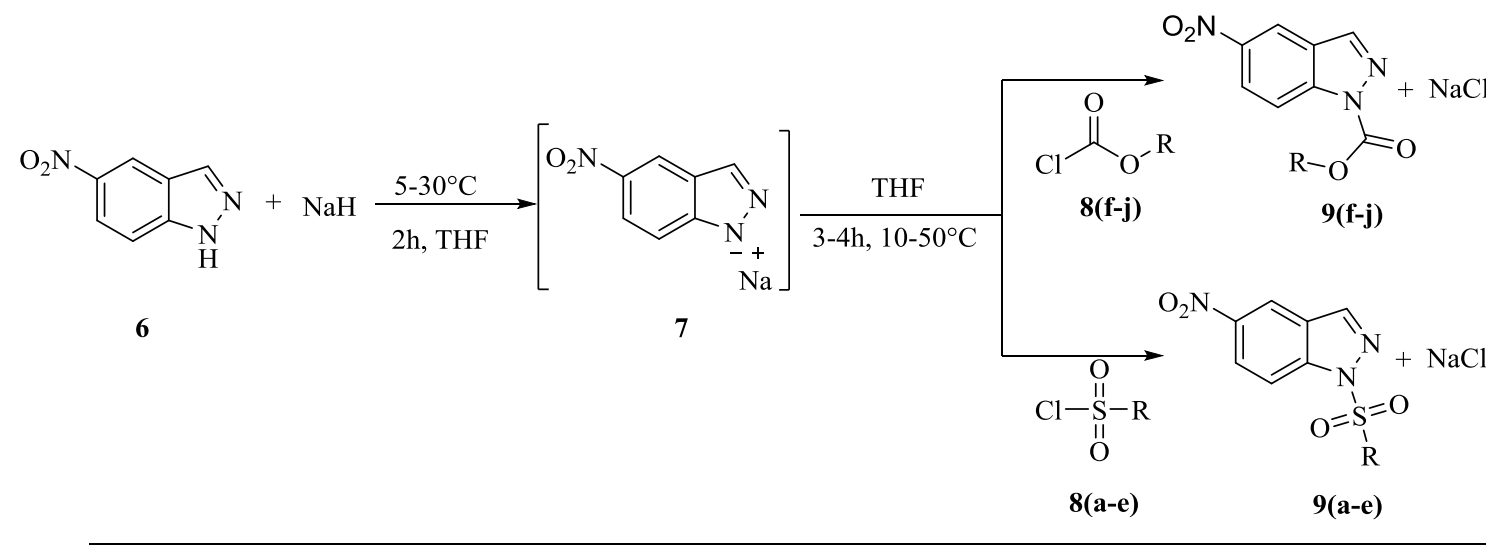

Compd.

Scheme 1. Synthesis of sulfonamide and carbamate derivatives of 5-nitroindazole.

All the newly synthesized compounds were characterized by IR, ${ }^{1} \mathrm{H}$ NMR, ${ }^{13} \mathrm{C}$ NMR and mass spectral data. IR absorption bands for $\mathrm{C}-\mathrm{H}, \mathrm{NO}_{2}, \mathrm{SO}_{2}$ and $\mathrm{C}-\mathrm{O}-\mathrm{C}$ were corresponded to 30913096, 1525-1527, 1345-1357 and 1034-1032 $\mathrm{cm}^{-1}$ respectively. In ${ }^{1} \mathrm{H}$ NMR spectra (400 MHz), aromatic protons resonated as multiplets in the range of 7.25-8.70 ppm. $-\mathrm{CH}_{2}$ protons of $9 \mathrm{f}$ resonated at $4.18 \mathrm{ppm}$ and other protons of the structures were observed in their expected regions. The ${ }^{13} \mathrm{C}$ NMR spectra were recorded for a few representative compounds and the data are given in the experimental part. The carbon chemical shifts values in the range of 150.4-150.9 ppm are assigned to carbamate derivatives $-\mathrm{C}=\mathrm{O} 9 \mathbf{9}-\mathbf{j}$. The LC-MS for a few title compounds were recorded and the presence of $\mathrm{M}^{+}$. at their respective $\mathrm{m} / \mathrm{z}$ values confirm the structures. The $\mathrm{C}, \mathrm{H}, \mathrm{N}$ analyses data were obtained for representative compounds and gave satisfactory values when compared to calculated values.

Antibacterial activity of the newly synthesized compounds was investigated at two different concentrations 100, $200 \mu \mathrm{g} / \mathrm{mL}$ using disc diffusion method against Gram positive bacteria such as Staphylococcus aureus and Lacto bacillus and Gram negative bacteria such as Pseudomonas florescensa and Escherichia coli and Ciprofloxacin was used as a standard drug. All the synthesized compounds $\mathbf{9}(\mathbf{a}-\mathbf{j})$ were found to exhibit good to moderate antibacterial activity against tested strains. A few of the compounds showed significant activity, where as 9a showed good activity against all the tested bacterial strains, 9d, $9 \mathbf{f}$ and $\mathbf{9 j}$ showed high activity against $S$. aureus, E. coli and $9 \mathbf{f}$ and $\mathbf{9 j}$ exhibited good activity against $S$. aureus, $P$. florescensa.

Antifungal activity of the title compounds was also screened against fungal pathogens such as Aspergillus niger and Penicillium chrysogenum by a disc-diffusion technique using standard drug, Miconazole. All the title compounds $\mathbf{9 a - j}$ exhibited good to moderate activity against tested fungal strains. The compounds $9 \mathbf{a}, \mathbf{9 d}, \mathbf{9 f}$ and $\mathbf{9 j}$ showed good activity, against all the two tested fungal strains. The results revealed that the fluorinated $\left(\mathrm{F} / \mathrm{CF}_{3}\right)$ compounds $(\mathbf{9 b}$ and $9 \mathbf{d})$ and nitro functionalized compounds such as $9 \mathbf{e}$, 9f showed a high degree of the zone of inhibition when compared to other functionalities present in the title compounds. 


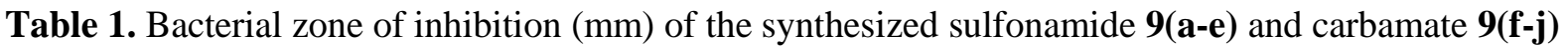
derivatives of 5-nitro- $1 H$-indazole.

\begin{tabular}{|c|c|c|c|c|c|c|c|c|}
\hline \multicolumn{9}{|c|}{ Bacterial culture and zone of inhibition in $\mathbf{~ m m}$} \\
\hline \multirow[t]{2}{*}{ Compd. } & \multicolumn{2}{|c|}{ S. aureus } & \multicolumn{2}{|c|}{ L. bacillus } & \multicolumn{2}{|c|}{ P. florescensa } & \multicolumn{2}{|c|}{ E. coli } \\
\hline & $\begin{array}{c}100 \\
\mu \mathrm{g} / \mathrm{mL}\end{array}$ & $\begin{array}{c}200 \\
\mu \mathrm{g} / \mathrm{mL}\end{array}$ & $\begin{array}{c}100 \\
\mu \mathrm{g} / \mathrm{mL}\end{array}$ & $\begin{array}{c}200 \\
\mu \mathrm{g} / \mathrm{mL}\end{array}$ & $\begin{array}{c}100 \\
\mu \mathrm{g} / \mathrm{mL}\end{array}$ & $200 \mu \mathrm{g} / \mathrm{mL}$ & $\begin{array}{c}100 \\
\mu \mathrm{g} / \mathrm{mL}\end{array}$ & $\begin{array}{c}200 \\
\mu \mathrm{g} / \mathrm{mL}\end{array}$ \\
\hline $9 a$ & 17.4 & 22.6 & 15.5 & 21.4 & 19.7 & 21.2 & 21.5 & 22.6 \\
\hline $9 b$ & 7.6 & 13.2 & 9.4 & 16.5 & 9.2 & 18.2 & 11.3 & 15.7 \\
\hline $9 c$ & 12.2 & 19.4 & 11.5 & 19.2 & 12.2 & 18.3 & 14.5 & 17.6 \\
\hline 9d & 15.7 & 21.3 & 19.1 & 21.7 & 19.3 & 20.5 & 13.6 & 18.2 \\
\hline $9 e$ & 8.4 & 12.9 & 6.8 & 13.2 & 8.9 & 12.7 & 11.6 & 13.2 \\
\hline 9f & 16.3 & 19.2 & 14.6 & 18.5 & 13.2 & 19.7 & 12.3 & 19.5 \\
\hline $9 g$ & 9.3 & 15.2 & 8.6 & 13.2 & 8.7 & 11.6 & 11.3 & 14.6 \\
\hline $9 \mathrm{~h}$ & 8.3 & 10.6 & 6.9 & 12.3 & 7.2 & 14.3 & 10.2 & 14.5 \\
\hline $9 \mathrm{i}$ & 11.2 & 13.5 & 11.4 & 15.9 & 11.3 & 13.3 & 12.3 & 15.3 \\
\hline $\mathbf{9 j}$ & 19.5 & 21.3 & 17.3 & 22.7 & 18.9 & 22.1 & 18.6 & 22.1 \\
\hline Ciprofloxacin* & 25 & & 26 & & 23 & & 24 & \\
\hline
\end{tabular}

*Ciprofloxacin was carried out at $100 \mu \mathrm{g} / \mathrm{mL}$.

The compounds 9a, 9d, 9f and 9j exhibited good activity against $A$. niger and $P$. chrysogenum. The zones of inhibitions of the mentioned compounds are almost close to the standard. The reason might be the presence of trichloromethyl group in $\mathbf{9 j}$, 4-chloro-3-nitrophenyl group in $\mathbf{9 a}$, $p$-nitrophenyl group in $\mathbf{9 e}, \mathbf{9 b}$ and 4-fluorophenyl group in $\mathbf{9 b}$ were responsible for the enhancement of the microbial activity. Slight variation in structure can have a very noticeable effect on the efficiency of these compounds in their bioactivity.

\section{Experimental}

\subsection{Chemistry}

Chemicals were procured from Sigma-Aldrich, Merck and used as such without further purification. All solvents used were spectroscopic and other physical studies were reagent grade and further purified by literature methods. Melting points (m.p) were determined using a calibrated thermometer by GUNA Digital melting point apparatus and are uncorrected. Infrared spectra (IR) were obtained on a Perkin-Elmer Model 281-B spectrophotometer. Samples were analyzed on potassium bromide $(\mathrm{KBr})$ disks. Absorptions were reported in wave numbers $\left(\mathrm{cm}^{-1}\right) .{ }^{1} \mathrm{H}$ and ${ }^{13} \mathrm{C}$ NMR spectra were recorded in $\mathrm{CDCl}_{3}$ on a Bruker AMX $400 \mathrm{MHz}$ spectrometer operating at $400 \mathrm{MHz}$ for proton and $100 \mathrm{MHz}$ for carbon. The ${ }^{1} \mathrm{H}$ and ${ }^{13} \mathrm{C}$ chemical shifts were expressed in parts per million (ppm) with reference to tetramethylsilane (TMS). LC-MS mass spectra were recorded on a Joel SX 102 DA/600 Mass spectrometer. Elemental analysis was performed on Thermo Finnigan FLASH EA 112 instrument.

Table 2. Fungal zone of inhibition ( $\mathrm{mm}$ ) of the synthesized sulfonamides $\mathbf{9}(\mathbf{a}-\mathbf{e})$ and carbamates $\mathbf{9}(\mathbf{f}-\mathbf{j})$ of 5-nitro- $1 H$-indazole. 


\begin{tabular}{ccccc}
\hline \multicolumn{5}{c}{ Fungal culture and zone of inhibition in mm } \\
\hline Compd. & \multicolumn{3}{c}{ A. niger } & \multicolumn{2}{c}{ P. chrysogenum } \\
\hline 9a & $100 \mu \mathrm{g} / \mathrm{mL}$ & $200 \mu \mathrm{g} / \mathrm{mL}$ & $100 \mu \mathrm{g} / \mathrm{mL}$ & 200 \\
& 19.4 & 21.8 & 18.6 & 21.5 \\
9b & 10.9 & 13.2 & 11.2 & 15.5 \\
9c & 13.2 & 17.4 & 10.5 & 16.5 \\
9d & 19.6 & 21.6 & 16.5 & 20.2 \\
9e & 7.4 & 14.4 & 7.8 & 12.2 \\
9f & 18.5 & 21.5 & 19.1 & 22.4 \\
9g & 9.3 & 13.9 & 8.6 & 13.8 \\
9h & 6.3 & 11.7 & 6.9 & 11.3 \\
9i & 11.3 & 15.5 & 10.2 & 13.7 \\
9j & 18.2 & 22.5 & 17.7 & 21.6 \\
Miconazole & $\mathbf{2 4}$ & & $\mathbf{2 5}$ & \\
\hline
\end{tabular}

*Miconazole was carried out at $100 \mu \mathrm{g} / \mathrm{mL}$.

\subsection{General Procedure}

To a stirred solution of 5-nitro-1H-indazole (6) $(0.001 \mathrm{~mol}, 252 \mathrm{mg})$ in $10 \mathrm{~mL}$ of THF, sodium hydride $(0.001 \mathrm{~mol})$ was added at $5-10^{\circ} \mathrm{C}$. The reaction mixture was slowly raised to $30^{\circ} \mathrm{C}$ and stirred for 2 h. 4-Chloro-3-nitrobenzenesulfonyl chloride (8a) $(0.001 \mathrm{~mol})$ in $10 \mathrm{~mL}$ of THF was added slowly at $10-15^{\circ} \mathrm{C}$. Later the reaction mixture was stirred at $40-50^{\circ} \mathrm{C}$ for $3 \mathrm{~h}$. The progress of the reaction was monitored by TLC. After completion of the reaction, the $\mathrm{NaCl}$ was filtered, and the filtrate was concentrated in a rota evaporator and the crude product was purified by silica gel column chromatography using hexane:ethlylacetate $(2: 1)$ as an eluent to get pure 1-(4-chloro-3nitrophenylsulfon-yl)-5-nitro-1H-indazole in $89 \%$ of yield. All the title compounds were synthesized by using same procedure. The structures of the newly synthesized new sulphonamide and carbamate derivatives $\mathbf{9}(\mathbf{a}-\mathbf{j})$ were confirmed by spectroscopic data.

\subsection{Spectral Data}

3.3.1. 1-(4-Chloro-3-nitrophenylsulfonyl)-5-nitro-1H-indazole (9a):<smiles>O=[N+]([O-])c1ccc(S(=O)(=O)c2ccc(Cl)c([N+](=O)[O-])c2)cc1</smiles>

Light Yellow solid, Yield: $89 \%$; Mol. Wt: 382.7, m.p: 260-262 ${ }^{0} \mathrm{C}$; IR ( $\left.\mathrm{cm}^{-1}, \mathrm{KBr}\right): 3091$ (-C-H), $1694(\mathrm{C}=\mathrm{C}), 1596(\mathrm{C}-\mathrm{C}), 1525\left(\mathrm{NO}_{2}\right), 1345(\mathrm{~S}=\mathrm{O}), 1296(\mathrm{C}-\mathrm{N}), 1056(\mathrm{C}-\mathrm{N}), 897(\mathrm{~N}-\mathrm{S})$ and $833(\mathrm{C}-$ $\mathrm{Cl}) ;{ }^{1} \mathrm{H}$ NMR $\left(400 \mathrm{MHz}, \mathrm{CDCl}_{3}\right): \delta 8.05-8.06(\mathrm{~d}, 1 \mathrm{H}, J=4 \mathrm{~Hz}, \mathrm{Ar}-\mathrm{H}), 8.08(\mathrm{~d}, 1 \mathrm{H}, J=2.8 \mathrm{~Hz}, \mathrm{Ar}-$ $\mathrm{H}), 8.21(\mathrm{~s}, 1 \mathrm{H}, \mathrm{Ar}-\mathrm{H}), 8.31(\mathrm{~d}, 1 \mathrm{H}, J=8.8 \mathrm{~Hz} \mathrm{Ar}-\mathrm{H}), 8.46(\mathrm{~d}, 1 \mathrm{H}, J=7.1 \mathrm{~Hz}, \mathrm{Ar}-\mathrm{H}), 8.67(\mathrm{~s}, 1 \mathrm{H}, \mathrm{Ar}-$ $\mathrm{H}), 8.8(\mathrm{~s}, 1 \mathrm{H}, \mathrm{Ar}-\mathrm{H}) ;{ }^{13} \mathrm{C}$ NMR $\left(100 \mathrm{MHz}, \mathrm{CDCl}_{3}\right): \delta 149.3,146.9,143.8,136.6,136.6,133.3,132.8$, 125.6, 124.3, 122.9, 118.6, 113.5; LCMS (\%): $\mathrm{m} / \mathrm{z} 382[\mathrm{M}]^{+}(100 \%)$. Elemental Analysis: calculated for $\mathrm{C}_{13} \mathrm{H}_{7} \mathrm{ClN}_{4} \mathrm{O}_{6} \mathrm{~S}$ C, 40.80; H, 1.84; N, 14.64; Found: C, 40.95, H,1.96, N, 14.72 . 


\subsubsection{1-(4-Flurophenyl sulfonyl)-5-nitro-1H-indazole (9b):}<smiles>O=[N+]([O-])c1ccc(S(=O)(=O)c2ccc(F)cc2)cc1</smiles>

Light Yellow solid, Yield: $88 \%$; Mol. Wt: 321.2, m.p: 210-212 ${ }^{0} \mathrm{C}$; IR ( $\left.\mathrm{cm}^{-1}, \mathrm{KBr}\right): 3095$ (-C-H), 1684 $(\mathrm{C}=\mathrm{C}), 1598(\mathrm{C}-\mathrm{C}), 1524\left(\mathrm{NO}_{2}\right), 1340(\mathrm{~S}=\mathrm{O}), 1290(\mathrm{C}-\mathrm{N}), 1054(\mathrm{C}-\mathrm{N}), 893(\mathrm{~N}-\mathrm{S})$ and $835(\mathrm{C}-\mathrm{F}) ;{ }^{1} \mathrm{H}$ NMR (400 MHz, CDCl $): \delta 8.85(\mathrm{~s}, 1 \mathrm{H}, \mathrm{Ar}-\mathrm{H}), 8.46(\mathrm{~d}, 1 \mathrm{H}, J=4 \mathrm{~Hz}, \mathrm{Ar}-\mathrm{H}), 8.33(\mathrm{~d}, 1 \mathrm{H}, J=8.7 \mathrm{~Hz}$ Ar-H), 8.23 (s,1H, Ar-H), 7.44-7.84 (m, 4H, Ar-H).

\subsubsection{1-(4-Bromophenyl sulfonyl)-5-nitro-1H-indazole (9c):}<smiles>O=[N+]([O-])c1ccc(S(=O)(=O)c2ccc(Br)cc2)cc1</smiles>

Light Yellow solid, Yield: $84 \%$; Mol. Wt: 382.1, m.p: 221-223우 $\mathrm{IR}\left(\mathrm{cm}^{-1}, \mathrm{KBr}\right): 3093$ (-C-H), 1695 $(\mathrm{C}=\mathrm{C}), 1599(\mathrm{C}-\mathrm{C}), 1523\left(\mathrm{NO}_{2}\right), 1342(\mathrm{~S}=\mathrm{O}), 1291(\mathrm{C}-\mathrm{N}), 1051(\mathrm{C}-\mathrm{N}), 891(\mathrm{~N}-\mathrm{S})$ and $836(\mathrm{C}-\mathrm{Br})$; ${ }^{1} \mathrm{H}$ NMR $\left(400 \mathrm{MHz}, \mathrm{CDCl}_{3}\right): \delta 7.03-8.07(\mathrm{~m}, 4 \mathrm{H}, \mathrm{Ar}-\mathrm{H}), 8.28(\mathrm{~s}, 1 \mathrm{H}, \mathrm{Ar}-\mathrm{H}), 8.38(\mathrm{~d}, 1 \mathrm{H}, J=8.5 \mathrm{~Hz}$ Ar-H), 8.75 (d, $1 \mathrm{H}, J=2.8 \mathrm{~Hz}$, Ar-H ), $8.86(\mathrm{~s}, 1 \mathrm{H}, \mathrm{Ar}-\mathrm{H})$.

\subsubsection{5-Nitro-1-(4-(trifluoromethyl) phenyl sulfonyl)-1H-indazole (9d):}<smiles>O=[N+]([O-])c1ccc(S(=O)(=O)c2ccc(C(F)(F)F)cc2)cc1</smiles>

Light Yellow solid, Yield: 73 \%; Mol. Wt: 371.29, m.p : 141-143 ${ }^{0} \mathrm{C}$; IR ( $\left.\mathrm{cm}^{-1}, \mathrm{KBr}\right): 3090$ (-C-H), 1691 (C=C), $1597(\mathrm{C}-\mathrm{C}), 1520\left(\mathrm{NO}_{2}\right), 1343(\mathrm{~S}=\mathrm{O}), 1294(\mathrm{C}-\mathrm{N}), 1053(\mathrm{C}-\mathrm{N}), 893(\mathrm{~N}-\mathrm{S})$ and $832(\mathrm{C}-\mathrm{F})$; ${ }^{1} \mathrm{H}$ NMR $\left(400 \mathrm{MHz}, \mathrm{CDCl}_{3}\right): \delta 7.77-7.78(\mathrm{~m}, 4 \mathrm{H}, \mathrm{Ar}-\mathrm{H}), 8.25(\mathrm{~s}, 1 \mathrm{H}, \mathrm{Ar}-\mathrm{H}), 8.36(\mathrm{~d}, 1 \mathrm{H}, J=4 \mathrm{~Hz} \mathrm{Ar}-$ $\mathrm{H}), 8.48(\mathrm{~d}, 1 \mathrm{H}, J=2 \mathrm{~Hz}, \mathrm{Ar}-\mathrm{H}), 8.65(\mathrm{~s}, 1 \mathrm{H}, \mathrm{Ar}-\mathrm{H})$. 


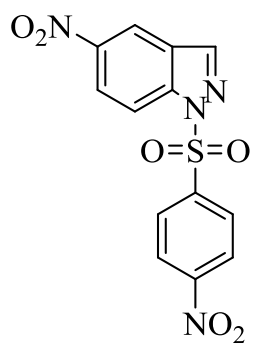

Light Yellow solid, Yield: $83 \%$; Mol. Wt: 348.2, m.p : 263-265 ${ }^{0}$; IR ( $\left.\mathrm{cm}^{-1}, \mathrm{KBr}\right): 3092(-\mathrm{C}-\mathrm{H})$, $1698(\mathrm{C}=\mathrm{C}), 1594(\mathrm{C}-\mathrm{C}), 1526\left(\mathrm{NO}_{2}\right), 1342(\mathrm{~S}=\mathrm{O}), 1293(\mathrm{C}-\mathrm{N}), 1055(\mathrm{C}-\mathrm{N})$ and $894(\mathrm{~N}-\mathrm{S}) ;{ }^{1} \mathrm{H}$ NMR $\left(400 \mathrm{MHz}, \mathrm{CDCl}_{3}\right): \delta 8.91(\mathrm{~s}, 1 \mathrm{H}, \mathrm{Ar}-\mathrm{H}), 8.66-8.67$ (d, $\left.1 \mathrm{H}, J=1.6 \mathrm{~Hz}, \mathrm{Ar}-\mathrm{H}\right), 8.55-8.56(\mathrm{~d}, 1 \mathrm{H}, J=$ $4 \mathrm{~Hz}$ Ar-H), 8.21 (s, 1H, Ar-H), 8.15-8.14 (m, 4 H, Ar-H).

3.3.6. 4 -Nitrobenzyl-5-nitro-1H-indazole-1-carboxylate (9f):<smiles>O=C(OCc1ccc([N+](=O)[O-])cc1)n1ncc2cc([N+](=O)[O-])ccc21</smiles>

Yield: 79 \%, Mol. Wt: 342.2, m.p. 180-182 ${ }^{\circ} \mathrm{C}$; IR ( $\left.\mathrm{cm}^{-1}, \mathrm{KBr}\right): 3090$ (-C-H), 1736 (C=O), 1610 $(\mathrm{C}=\mathrm{N}), 1527\left(\mathrm{NO}_{2}\right), 1434(\mathrm{C}-\mathrm{N}), 1330(\mathrm{C}-\mathrm{C})$ and $1034(\mathrm{C}-\mathrm{O}-\mathrm{C}) ;{ }^{1} \mathrm{H}$ NMR $\left(400 \mathrm{MHz}, \mathrm{CDCl}_{3}\right): \delta$ 8.89 (s, $1 \mathrm{H}, \mathrm{Ar}-\mathrm{H}), 8.66(\mathrm{~d}, 1 \mathrm{H}, J=2.0 \mathrm{~Hz}, \mathrm{Ar}-\mathrm{H}), 8.41(\mathrm{~s}, 1 \mathrm{H}, \mathrm{Ar}-\mathrm{H}), 8.29$ (d, $1 \mathrm{H}, J=8.8 \mathrm{~Hz}$ Ar$\mathrm{H}), 7.65(\mathrm{~m}, 4 \mathrm{H}, \mathrm{Ar}-\mathrm{H}), 5.17\left(\mathrm{~s}, 2 \mathrm{H},\left(\mathrm{CH}_{2}\right)\right.$.

3.3.7. Ethyl-5-nitro-1H-indazole-1-carboxylate $(\mathbf{9 g})$ :<smiles>CCOC(=O)n1ncc2cc([N+](=O)[O-])ccc21</smiles>

Brown solid, Yield: $69 \%$, Mol. Wt: 235.06, m.p. 146-148 ${ }^{0} \mathrm{C}$; IR $\left(\mathrm{cm}^{-1}, \mathrm{KBr}\right): 3094(-\mathrm{C}-\mathrm{H}), 1737$ $(\mathrm{C}=\mathrm{O}), 1607(\mathrm{C}=\mathrm{N}), 1521\left(\mathrm{NO}_{2}\right), 1441(\mathrm{C}-\mathrm{N}), 1332(\mathrm{C}-\mathrm{C})$ and $1032(\mathrm{C}-\mathrm{O}-\mathrm{C}) ;{ }^{1} \mathrm{H}$ NMR (400 MHz, $\left.\mathrm{CDCl}_{3}\right): \delta 8.70(\mathrm{~d}, 1 \mathrm{H}, J=4 \mathrm{~Hz}, \mathrm{Ar}-\mathrm{H}), 8.45(\mathrm{~d}, 1 \mathrm{H}, J=7.2 \mathrm{~Hz}, \mathrm{Ar}-\mathrm{H}), 8.43(\mathrm{~s}, 1 \mathrm{H}, \mathrm{Ar}-\mathrm{H}), 8.27$ $(\mathrm{d}, 1 \mathrm{H}, J=8.7 \mathrm{~Hz} \mathrm{Ar}-\mathrm{H}), 4.37\left(\mathrm{q}, 2 \mathrm{H},\left(\underline{\mathrm{CH}}_{2}-\mathrm{CH}_{3}\right)\right), 1.29\left(\mathrm{t}, 3 \mathrm{H}, J=4 \mathrm{~Hz}, \mathrm{CH}_{2}-\underline{\mathrm{CH}}_{3}\right)$. 
<smiles>CC(C)COC(=O)n1ncc2cc([N+](=O)[O-])ccc21</smiles>

Brown solid, Yield: $77 \%$, Mol. Wt: 263.2, m.p. 191-193 ${ }^{0} \mathrm{C}$; IR $\left(\mathrm{cm}^{-1}, \mathrm{KBr}\right): 3096$ (-C-H), 1730 $(\mathrm{C}=\mathrm{O}), 1609(\mathrm{C}=\mathrm{N}), 1524\left(\mathrm{NO}_{2}\right), 1446(\mathrm{C}-\mathrm{N}), 1338(\mathrm{C}-\mathrm{C})$ and $1037(\mathrm{C}-\mathrm{O}-\mathrm{C}) ;{ }^{1} \mathrm{H}$ NMR $(400 \mathrm{MHz}$, $\left.\mathrm{CDCl}_{3}\right): \delta 8.73(\mathrm{~s}, 1 \mathrm{H}, \mathrm{Ar}-\mathrm{H}), 8.46(\mathrm{~s}, 1 \mathrm{H}, \mathrm{Ar}-\mathrm{H}), 8.43(\mathrm{~d}, 1 \mathrm{H}, J=2 \mathrm{~Hz}, \mathrm{Ar}-\mathrm{H}), 8.38(\mathrm{~d}, 1 \mathrm{H}, J=8.4$ $\mathrm{Hz} \mathrm{Ar}-\mathrm{H}), 3.75\left(\mathrm{~d}, 2 \mathrm{H}, J=4 \mathrm{~Hz} \mathrm{CH}\right.$ ), $1.97(\mathrm{~m}, 1 \mathrm{H}, \mathrm{CH}), 0.87$ (d, $\left.6 \mathrm{H}, J=6.8 \mathrm{~Hz}\left(\mathrm{CH}_{3}\right)_{2}\right)$.

3.3.9. Methyl-5-nitro-1H-indazole-1-carboxylate $(\mathbf{9 i}):^{28}$<smiles>COC(=O)n1ncc2cc([N+](=O)[O-])ccc21</smiles>

Brown solid, Yield: $85 \%$, Mol. Wt: 221.04, m.p. 171-173 ${ }^{0}$; IR ( $\left.\mathrm{cm}^{-1}, \mathrm{KBr}\right): 3093(-\mathrm{C}-\mathrm{H}), 1734$ $(\mathrm{C}=\mathrm{O}), 1605(\mathrm{C}=\mathrm{N}), 1522\left(\mathrm{NO}_{2}\right), 1443(\mathrm{C}-\mathrm{N}), 1335(\mathrm{C}-\mathrm{C})$ and $1036(\mathrm{C}-\mathrm{O}-\mathrm{C}) ;{ }^{1} \mathrm{H}$ NMR (400 MHz, $\left.\mathrm{CDCl}_{3}\right): \delta 8.71(\mathrm{~s}, 1 \mathrm{H}, \mathrm{Ar}-\mathrm{H}), 8.45(\mathrm{~d}, 1 \mathrm{H}, J=4 \mathrm{~Hz}, \mathrm{Ar}-\mathrm{H}), 8.43(\mathrm{~s}, 1 \mathrm{H}, \mathrm{Ar}-\mathrm{H}), 8.36(\mathrm{~d}, 1 \mathrm{H}, J=7.8$ $\mathrm{Hz} \mathrm{Ar}-\mathrm{H}), 4.18\left(\mathrm{~s}, 3 \mathrm{H},\left(\mathrm{CH}_{3}\right)\right) ;{ }^{13} \mathrm{C}$ NMR $\left(100 \mathrm{MHz}, \mathrm{CDCl}_{3}\right): \delta 150.7,144.8,142.2,140.9,125.6$, 124.3, 118.1, 115.7, 55.2; LCMS (\%): $m / z$ 221.1(100\%) [M] ${ }^{+}$; Chemical Formula: $\mathrm{C}_{9} \mathrm{H}_{7} \mathrm{~N}_{3} \mathrm{O}_{4}$, Elemantal Analysis: C, 46.68; H, 5.14; N, 6.80; Found: C, 47.85, H,5.45, N, 6.48.

\subsubsection{2,2,2-Trichloroethyl-5-nitro-1H-indazole-1-carboxylate (9j):}<smiles>O=C(OCC(Cl)(Cl)Cl)n1ncc2cc([N+](=O)[O-])ccc21</smiles>

Brown solid, Yield: $76 \%$, Mol. Wt: 338.5, m.p. 156-158 ${ }^{0} \mathrm{C}$; IR $\left(\mathrm{cm}^{-1}, \mathrm{KBr}\right)$ : $3091(-\mathrm{C}-\mathrm{H}), 1738$ $\left.(\mathrm{C}=\mathrm{O}), 1601(\mathrm{C}=\mathrm{N}), 1528\left(\mathrm{NO}_{2}\right), 1442 \mathrm{C}-\mathrm{N}\right), 1336(\mathrm{C}-\mathrm{C}), 1035(\mathrm{C}-\mathrm{O}-\mathrm{C})$ and $830(\mathrm{C}-\mathrm{Cl}) ;{ }^{1} \mathrm{H}$ NMR $\left(400 \mathrm{MHz}, \mathrm{CDCl}_{3}\right): \delta 8.74(\mathrm{~s}, 1 \mathrm{H}, \mathrm{Ar}-\mathrm{H}), 8.54(\mathrm{~d}, 1 \mathrm{H}, J=4 \mathrm{~Hz}, \mathrm{Ar}-\mathrm{H}), 8.43$ (s, 1H, Ar-H ), 8.27 (d, $1 \mathrm{H}, J=8.9 \mathrm{~Hz} \mathrm{Ar}-\mathrm{H}), 4.90\left(\mathrm{~s}, 3 \mathrm{H},\left(\mathrm{CH}_{2}\right)\right.$.

\subsection{Antimicrobial Activity}

\subsubsection{Antibacterial Activity}

All the newly synthesized sulfonamides and carbamates of 5-nitro-1H-indazole 9a-e and 9f-j were screened against two Gram positive bacteria such as Staphylococcus aureus and L. bacillus and two Gram negative bacteria such as $P$. florescensa and $E$. coli by agar well diffusion method. ${ }^{29}$ The synthesized compounds were screened at two concentrations of 100 and $200 \mu \mathrm{g} / \mathrm{mL}$. Centrifuged pellets of bacteria from $24 \mathrm{~h}$ old culture containing approximately $10^{4}-10^{6}$ colony forming unit (CFU) per $\mathrm{mL}$ was spread on the surface of Muller Hinton Agar (MHA) plates. Nutrient agar medium prepared by suspended nutrient agar $28 \mathrm{~g}$ in 1 liter of distilled water, autoclaved and cooled to $45^{\circ} \mathrm{C}$, then it was seeded with $15 \mathrm{~mL}$ of prepared inocula to have $10^{6} \mathrm{CFU} / \mathrm{mL}$. Petri dishes were prepared by pouring $10 \mathrm{~mL}$ of seeded nutrient agar. Wells was created in medium with the help of a sterile 
metallic borer and test solution was added. Experimental plates were incubated for $24 \mathrm{~h}$ at $37^{\circ} \mathrm{C}$ and antibacterial activity was defined as the diameter $(\mathrm{mm})$ of the clear inhibition zone formed around the well. Ciprofloxacin was used as a standard for antibacterial assay. The zone of inhibition of the tested solution was compared with standard and the results are given in bacterial zone of inhibition $(\mathrm{mm})$.

\subsubsection{Antifungal Activity}

Antifungal activity of sulfonamide (9a-e) and carbamate (9f-j) derivatives of 5-nitro- $1 \mathrm{H}$ indazole (1) was tested against Aspergillus niger and Penicillium chrysogenum by poison plate technique ${ }^{30}$ The compounds were dissolved in DMSO before mixing with potato dextrose agar (PDA). The final concentration of the compounds in the medium was fixed at 100 and $200 \mu \mathrm{g} / \mathrm{mL}$. Fungi were incubated in PDA at $25 \pm 1{ }^{\circ} \mathrm{C}$ for 5 days to get new mycelium for antifungal assay, and then a mycelia disk of approximately $0.60 \mathrm{~cm}$ diameter was cut from the culture medium and picked up with a sterilized inoculation needle and inoculated in the centre of the PDA plate. The inoculated plates were incubated at $25 \pm 1{ }^{\circ} \mathrm{C}$ for 5 days. DMSO solvent was added as negative control to determine possible inhibitory activity of the solvent, while miconazole was used as a positive control. For each treatment, three replicates were carried out and the mean of the diameter of the inhibition zones was calculated as fungal zone of inhibition (mm).

\section{Conclusion}

Synthesis of a series of new sulphonamide and carbamate derivatives of 5-nitroindazole was accomplished with high yields. Their structures were established by spectral data. Synthesized compounds screened for biological studies which displayed moderate to good activities. The results revealed that the fluorinated $\left(\mathrm{F} / \mathrm{CF}_{3}\right)$ compounds in $\mathbf{9 b}$ and $\mathbf{9 d}$ and nitro functionalized compounds such as $9 \mathrm{e}, \mathbf{9 g}$ showed high degree of the zone of inhibition as compared with other functionalities present in title compounds. The antibacterial activity of these compounds was also compared with that of commercial antibiotic, Ciprofloxacin.

\section{Acknowledgement}

The Authors would like to thank DST-PURSE Centre, Sri Venkateswara University for evaluating the antimicrobial activity.

\section{Supporting Information}

Supporting information accompanies this paper on http://www.acgpubs.org/OC

\section{ORCID}

K. Pushpa Kumar: 0000-0002-4042-3396

Pandreti Vedavathi: 0000-0002-9785-3709

K. Venkata Subbaiah: 0000-0001-7315-3575

D. Venkata Ramana Reddy: 0000-0003-1701-7231

C. Naga Raju: 0000-0001-6566-2118

\section{References}

[1] (a) Stadlbauer, W,; In Houben-Weyl, Methoden der Organischen Chemi: Indazole Benzopyrazole); Schaumann, E.; Ed.; Georg-Thieme-Verlag Stuttgart: New York, 1994, Vol. E8b, Hetarenes III/2, pp. 764-864; (b) Stadlbauer W. In Science of Synthesis: Indazoles, Neier, R., Ed.; Georg-Thieme-Verlag Stuttgart: New York. 2002, Vol. 2.12.4 (Hetarenes), pp. 227-324.

[2] North, M,; C. Amines and amides contemp. Org. Synth. 1994, l, 475 c. 
[3] Pradhan, K. J,; Variyar, P. S,; Bandekar, J. R,; Lebensm, W. U,; Synthesis, structure analysis and antibacterial activity of $N$-[5-dehydroabietyl-[1,3,4]thiadiazol-2-yl]-aromatic amide derivatives. Technol. 1999,32, 121

[4] Holban, M,; Şunel, V,; Popa, M,; Lionte, C,; Synthesis and study of new indazole derivatives with potential pharmacological activity. Cell. Chem. Technol. 2011, 45, 191.

[5] Strupczewski, J. T,; Helsey, G. C,; Glamkowski, E. J,; Chiang, Y,; Bordeau, K. J,; Nemoto, P. A., Tegele, J, Synthesis and study of new indazole derivatives with potential pharmacological activity. Chem. Abstr. 1998, 129, 122674-a.

[6] Ikeda, Y.; Takano, N.; Matsushita, H.; Shiraki, Y.; Koide, T.; Nagashima, R.; Fujimura, Y.; Shindo, M.; Suzuki, S.; Iwasaki, T. Chemistry and Biology of indoles and Indazoles: A mini-review. Arzneim.Forsch. 1979, 29, 511-520.

[7] Picciola, G.; Ravenna, F.; Carenini, G.; Gentili, P.; Riva, M. Synthesis and structural characterization of 1- and 2-substituted indazoles: Ester and carboxylic acid deriva tives. Farmaco Ed. Sci. 1981, 36, 1037-1056.

[8] The Merck Index, 12th edition; Budavari, S., Ed,; Merck \& Co.: Rahway, New Jersey. 1996.

[9] Mosti, L.; Menozzi, G.; Fossa, P.; Schenone, P.; Lampa, E.; Parrillo, C.; D'Amisco, M Rossi, F. Synthesis and antibacterial activity of Substituted-1- (1tosyl/methylsulphonyl-1H-indol-2-yl) methyl)$1 H$-Indazoles. Farmaco. 1992, 47, 567-584.

[10] Andronati, S.; Sava, V.; Makan, S.; Kolodeev, G Synthesis, characterization and evaluation of anticancer activity of some 7-(substituted benzylidene)-3-Aryl-2, 3, 4, 5, 6, 7-hexahydro indazol-1Yl(pyridin-4-Yl)methanones. Pharmazie. 1999, 54, 99-101.

[11] Kharitonov, V. G.; Sharma, V. S.; Magde, D.; Koesling, D. Characterization of a Novel Type of Endogenous Activator of Soluble Guanylyl Cyclase. Biochemistry 1999, 38, 10699-10706.

[12] Morie, T.; Harada, H.; Kato, S. Synthesis and Antimicrobial Activity of Novel mono- and bis- $\alpha-$ Aminophosphonate Derivatives.Synth. Commun. 1997, 27, 559-566; (b) Bermudez, J.; Fake, C. S.; Joiner, G. F.; Joiner, K. A.; King, F. D.; Miner, W. D.; Sanger, G. J. J. Hetercyclic chemistry in Drug Discovery. Med. Chem. 1990, 33, 1924-1929.

[13] Bouillon, I.; Zajicek, J.; Pudelova, N.; Krchnak, V.; J. Synthesis and study of new indazole derivatives with potential pharmacological activity. Org. Chem., 2008, 21, 9027

[14] Shimada, I.; Maeno, K.; Kazuta, K.; Kubota, H.; Kimizuka, T.; Kimura, Y.; Hatanaka, K.; Naitou, Y.; Wanibuchi, F.; Sakamato, S.; Tsukamato, S.; Bioorg. Med. Chem., 2008, 16, 1966.

[15] Moise, M.; Şunel, V.; Profire, L.; Popa, M.; Desbrieres, J.; Peptu, C.; Bul. Inst. Polit. Iași, S.IIc, 2009, 55, (59), 57.

[16] Petri, W.A.; Good man\& Gilman's The Pharmacological Basis of Therapeutics, 10th ed.; J.G.; Hardman, L.E. Limbird,A. G. Gilman, Eds.; McGraw-Hill: New York, 2001; pp 1171- 1179.

[17] Berger, K.; Petersen, B.; Pfaue, B.H. Persistence of drugs occurring in liquid manure in the food chain. Arch. Lebensmittelhyg. 1986, 37, 85-108.

[18] Thornber, C.W.; Isosterism and molecular modification in drug design. Chem. Soc Rev. 1979, 8, 563580 .

[19] Ogden, R.C.; Flexner, C.W. Protease inhibitors in AIDS therapy; New York, U.S.A: Marcel Dekker; 2001.

[20] Nishimori, I.; Vullo, D.; Innocenti, A.; Scozzafava, A.; Mastrolorenz, A.; Supuran, C.T. Carbonic anhydrase inhibitors: Inhibition of the transmembrane isozyme XIV with sulfonamides. Bioorg. Med. Chem. Lett. 2005, 15, 3828-3833.

[21] Li, J.J.; Anderson, D.; Burton, E.G.; Cogburn, J.N.; Collins, J.T.; Garland, D.J.; Gregory, S.A.; Huang, H.C.; Isakson, P.C.; Koboldt, C.M.; Logusch, E.W.; Norton, M.B.; Perkins, W.E.; Reinhard, E.J.; Seibert, K.; Veenhuizem, A.W.; Zang, Y.; Reitz, D.B. 1,2-Diarylcyclopentenes as selective cyclooxygenase- 2 inhibitors and orally active anti-inflammatory agents. J. Med. Chem. 1995, 38, 45704578.

[22] Boyd, A.E.; Sulfonylurea receptors, ion channels, and fruit flies. Diabetes 1988, 37, 847-850

[23] (a) Angela, T.S.; Andrea, C.; S. Protease inhibitors of the sulfonamide type: anticancer, antiinflammatory, and antiviral agents. Med. Res. Rev. 2003, 23, 535-538..; (b) Gokcen, T.; Al, M.; Topal, M.; Gulcin, İ.; Ozturk, T.; Goren A.C. Synthesis of some natural sulphonamide derivatives as carbonic anhydrase inhibitors. Org. Commun. 2017, 10, 15-23.

[24] Folkmann, M.; Lund, F.G. Acyloxymethyl carbonochloridates. New intermediates in prodrug synthesis. Synthesis. 1990, 1159-1166.

[25] Warrass, R.; Weismuller, K.H.; Jung, G. Cyclic oligocarbamates. Tetrahedron Lett. 1998, 39, 27152716. 
[26] Graca, M.; do Rosario, V.E.; Jim, I.; Luis, C.; Rui, M. A carbamate-based approach to primaquine prodrugs: Antimalarial activity, chemical stability and enzymatic activation. Bioorg. Med. Chem. 2012, 20, 886-892.

[27] Nakamura, Takeki. Silvedr halide photographic material usin g novel photographically useful groupreleasing compound. Jpn Kokai Tokkyo Koho 1989, JP 01026842 A 19890130.

[28] Kalcic, Igor.; Zovko, Marijana.; Jadrijevic-Mladar Takac, Milena.; Zorc, Br; Butula.; Ivan. Synthesis and reactions of some azolecarboxylic acid derivatives. Croatica Chem. Acta 2003, 76(3), 217-228.

[29] Kazmi, S.U.; Ali, S.N.; Jamal, S.A.; Atta-ur-Rehman, J.Pharm.Sci., 1991, 4, 113-118.

[30] Atta-ur-Rehman Choudhary, M.I.; Thomson, W.J. Bioassay Techniques for Drug Development, Harwood Academic Publishers., Amsterdam, 2001.

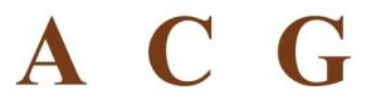

publications

(C) 2017 ACG Publications 